\title{
Ammonia Concentrations of Musts of Different Grape Cultivars and Vineyards in the Stellenbosch Area
}

\author{
C. S. OUGH ${ }^{\mathrm{a}}$ AND ABRAHAM KRIEL ${ }^{\mathrm{b}}$ \\ a Dept. Viticulture and Enology, University of California, Davis 95616 USA;
b Stellenbosch Farmers' Winery, Stellenbosch 7600, South Africa.
}

Date submitted: August 1984.

Date accepted: January 1985.
Key words: Ammonia, concentration, must, grape, cultivar.

\begin{abstract}
The ammonia concentrations of a large number of loads of grapes from different farms and of different cultivars were measured. Varietal differences were noted and statistical verifications made. Nine different cultivars were investigated. The average mean ammonia values for the cultivars varied from 54,9 to $148,1 \mathrm{mg} / \ell$. Where enough loads from individual vineyards or growers were available, statistical differences were demonstrated. The conditions, treatment, and soil of the vines were shown to be reflected in the extremes by the juice ammonia concentrations. The use of these data is suggested as a means of assessing the vineyard's relative worth for winemaking purposes.
\end{abstract}

There is relatively little real information on the ammonia content of grapes in relation to the cultivar of grapes. Ough (1969) found in California that ammonia content varied with cultivar but did not have enough samples for any definitive evaluations. The ammonia generally decreases with increasing maturity. The warmer the climate, the greater the loss. Puissant et al. (1960) noted that in poorer years (wet and cold) the ammonia content was higher in grapes in France.

It is common practice to add ammonia, either as diammoium phosphate or ammonium sulfate, to wines low in nitrogen to improve yeast growth and fermentation and to prevent hydrogen sulfide formation (Vos et al. 1979). This is seldom done in Calfornia but is not uncommon in other areas of the world where soils are poorer or improperly fertilized. However, Bell et al. (1979) showed that even very fertile soil depleted of nitrogen by leaching with grasses and having no nitrogen added would, after a period of time, yield grapes so low in nitrogen as to be very difficult to ferment properly and yield wine of lesser quality. Agenbach (1977) as well as Ough \& Kunkee (1968) showed the relationship of nitrogen source to fermentation efficiency.

The ammonia content of juice is easy to determine with the use of the ammonia electrode (McWilliam \& Ough, 1974).

This study was done to determine the average ammonia content in the grapes of the various cultivars most commonly grown in the Stellenbosch area. The value of the data is severalfold: it gives an indication of the nitrogen status of the vine and the fermentation properties of the juice and some indication of the worth of grapes from a specific cultivar and vineyard for the production of dry table wine. It has been shown that the fruity fermentation esters increase with increased nutrient status of the grape, and the fusel oils decrease (Ough \& Bell, 1980; and Ough \& Lee, 1981). This was also shown by Vos et al. (1979) for the addition of diammonium phosphate to wines low in free amino acids.

\section{MATERIALS AND METHODS}

Samples of juice taken for must analysis from trucks and gondolas arriving at the winery were analysed for ammonia. Sufficient loads were sampled to get statistical evaluations of the results. Each sample was identified according to the vineyard. The samples of the loads represented a reasonable sampling of each vineyard. All the loads from a specific vineyard were not necessarily picked in one day; hence, some maturity variation is included. The maturity was that which was acceptable to the winery. The ${ }^{\circ}$ Brix would vary from less than $19^{\circ}$ to over $26^{\circ}$. One hundred $\mathrm{ml}$ of each sample was made basic $(10 \mathrm{ml} 50 \% \mathrm{NaOH})$ to about $\mathrm{pH} 11.0$; while being stirred the sample was read immediately on an Orion Digital Ionanalyzer (Model 801A) using an Orion ammonia electrode. A standard curve was made using ammorium sulfate. Readings were compared to the standard curve. Samples were all read at a room temperature of $20^{\circ} \mathrm{C}$ $\pm 1{ }^{\circ} \mathrm{C}$.

All statistical analyses were performed in the standard way (Snedecor, 1956). Vineyards were compared statistically by analysis of variance. Only those with five or more loads of grapes measured were used. In comparing cultivars data from all the loads were used, except for Chenin blanc (Steen), where there were more than sufficient data.

The error in the actual measurement is very low. Table 1 shows the standard deviation on nine different musts, each measured three times. The coefficient error of the gondolas and trucks would be expected to be larger (Berg \& Marsh, 1954). Variations in the vineyard would also be larger than this (Amerine \& Roessler, 1958).

TABLE 1

Repeatablity of the ammonia measurements on individual samples of juice

\begin{tabular}{|c|c|c|c|}
\hline $\begin{array}{l}\text { Samples } \\
\text { Number }\end{array}$ & $\begin{array}{l}\text { Repetitions of same } \\
\text { Samples }\left(\mathrm{mg} \mathrm{NH}_{3} / \ell\right)\end{array}$ & $\mathrm{S}$ & $\mathrm{CV} \%$ \\
\hline 1 & $87,84,81$ & \pm 3.00 & \pm 3.57 \\
\hline 2 & $55,54,55$ & \pm 0.58 & \pm 1.06 \\
\hline 3 & $79,79,79$ & \pm 0.00 & 0.00 \\
\hline 4 & $73,69,73$ & \pm 2.31 & \pm 3.22 \\
\hline 5 & $58,57,55$ & \pm 1.53 & \pm 2.70 \\
\hline 6 & $60,60,61$ & \pm 0.58 & \pm 0.96 \\
\hline 7 & $62,61,63$ & \pm 1.00 & \pm 0.16 \\
\hline 8 & $57,59,57$ & \pm 1.15 & \pm 2.00 \\
\hline 9 & $79,79,79$ & 0.00 & 0.00 \\
\hline
\end{tabular}

Average Coefficient of Variation $= \pm 1.52$

S. Afr. J. Enol. Vitic., Vol. 6. No. 11985 
Estimates of the vine vigour, crop load, soil depth, nitrogen fertilizer applications and other viticultural facts were obtained on a number of the vineyards measured by actual observation and consultation with the grower.

\section{RESULT AND DISCUSSION}

The differences in the ammonia content of the different cultivars evaluated are shown in Table 2 . The cultivars are arranged according to decreasing ammonia content. There is an almost threefold difference in the mean values between the cultivar with the lowest ammonia value, Muscat d' Alexandrie and that with the highest, Pinotage. In this instance nine cultivars could be statistically sorted into six different groups despite the large standard deviations. The between vineyard deviations may be attributed to conditions of soil, moisture, and vineyard practices. Vineyard variations, due to maturity changes over the sampling period, may account for some of the standard deviations (Ough \& Singleton, 1968).

The individual cultivars were analysed on a per vineyard block basis. For Pinotage four growers brought in five or more loads which were sampled and analysed. The data are shown in Table 3. The $\mathrm{NH}_{3}$ content of the grapes on the four vineyards could statistically be divided into three separate groups. Table 4 gives the ammonia values for six vineyards with five or more loads for Cinsaut. Four separate groups could be statistically shown. The Cabernet Sauvignon juice ammonia values are given in Table 5 for 10 vineyards. There were only five statistically different groups in this case. Clairette blanche vineyards (9) showed the least variations of all the cultivars, Table 6 . They could only be put into two significantly different groups, even though the number of loads per vineyard was relatively high and the standard deviations were not excessive. What is rather odd is the very definite and clear break between the two groups with no overlap of statistically different mean values. The Chenin blanc (Steen) vineyards, Table 7, with five or more loads numbered 16. These could be statistically broken down into six significantly different groups. The distribution of vineyard mean values for these cultivars appears fairly normal compared to the odd distribution for Clairette blanche.

These data show, despite the rather large standard deviation of the vineyard loads from individual growers, that it was possible to differentiate between certain growers.

To determine if there was any correlation between the high or low ammonia values and the viticultural aspects of the vineyard, those growers who had mean ammonia values more than one half a standard deviation for $\mathrm{NH}_{3}$ above the mean for the cultivar, and those with mean values for $\mathrm{NH}_{3}$ one half a standard deviation below, are listed in Tables 8 and 9 respectively. The significant viticultural aspects are compared. In these cases data are used if three or more loads were sampled for the cultivar.

The subjective estimation of the vine condition for the high ammonia vineyards gave eight in good condition, 10 in average condition and none judged poor. In the low ammonia group there was one judged good, 14 judged average and nine judged poor. Most of the good vine condition vineyards were under 12 years old, with the average condition ones of intermediate age, and the poor
TABLE 2

Statistical data on all cultivars measured for ammonia $(\mathrm{mg} / \ell)$

\begin{tabular}{l|c|c|c|c}
\hline \multicolumn{1}{c|}{ Cultivar } & $\begin{array}{c}\text { Number } \\
\text { of }^{1} \text { loads }\end{array}$ & \multicolumn{1}{c|}{$\overline{\mathrm{X}}^{3}$} & $\mathrm{~S}$ & $\mathrm{CV} \%$ \\
\hline Pinotage & 45 & $148.1^{\mathrm{a}}$ & \pm 31.1 & $\pm 21.0 \%$ \\
Cinsaut & 90 & $107.0^{\mathrm{b}}$ & \pm 23.8 & $\pm 22.3 \%$ \\
Cabernet Sauvignon & 115 & $104.7^{\mathrm{b}}$ & \pm 32.4 & $\pm 31.0 \%$ \\
South African Riesling & 24 & $92.0^{\mathrm{c}}$ & \pm 33.5 & $\pm 36.4 \%$ \\
Colombard & 19 & $84.8^{\mathrm{d}}$ & \pm 26.2 & $\pm 30.9 \%$ \\
Shiraz & 18 & $72.2^{\mathrm{e}}$ & \pm 18.5 & $\pm 25.6 \%$ \\
Clairette blanche & 106 & $60.6^{\mathrm{f}}$ & \pm 14.1 & $\pm 23.3 \%$ \\
Chenin blanc (Steen) & 208 & $57.5^{\mathrm{f}}$ & \pm 18.6 & $\pm 32.3 \%$ \\
Muscat d' Alexandrie & 208 & & & \\
(Hanepoot) & 33 & $54.9^{\mathrm{f}}$ & \pm 20.1 & $\pm 36.6 \%$ \\
\hline
\end{tabular}

${ }^{1}$ Data from all loads used.

${ }^{2}$ Exception to ${ }^{1}$; only data from vineyards delivering 5 or more loads of this cultivar were used. (Mean Variation insignificant).

${ }^{3}$ Those $\bar{x}$ values with different superscripts are significantly different using $\operatorname{LSD}_{.05}=7.7$.

TABLE 3

Ammonia content $(\mathrm{mg} / \ell)$ of Pinotage grapes from four different vineyards

\begin{tabular}{c|c|c|c}
\hline $\begin{array}{c}\text { Grower } \\
\text { (Vineyard) }\end{array}$ & Number of loads & $\overline{\mathrm{X}}$ & $\mathrm{S}$ \\
\hline 1 & 9 & $175.8^{\mathrm{a}}$ & \pm 14.2 \\
2 & 11 & $167.5^{\mathrm{a}}$ & \pm 12.6 \\
3 & 7 & $150.9^{\mathrm{b}}$ & \pm 16.1 \\
4 & 7 & $109.9^{\mathrm{c}}$ & \pm 13.3 \\
\hline
\end{tabular}

Those $\overline{\mathrm{x}}$ values with different superscripts are significantly different using $\operatorname{LSD}_{.05}=13.67$.

TABLE 4

Ammonia content $(\mathrm{mg} / \ell)$ of Cinsaut grapes from six different vineyards

\begin{tabular}{c|c|c|c}
\hline $\begin{array}{c}\text { Grower } \\
\text { (Vineyard) }\end{array}$ & Number of loads & $\overline{\mathrm{X}}$ & $\mathrm{S}$ \\
\hline 7 & 5 & $135.4^{\mathrm{a}}$ & \pm 6.0 \\
3 & 29 & $120.2^{\mathrm{b}}$ & \pm 18.2 \\
8 & 10 & $116.2^{\mathrm{b}}$ & \pm 13.0 \\
9 & 7 & $108.9^{\mathrm{b}, \mathrm{c}}$ & \pm 24.6 \\
4 & 6 & $98.5^{\mathrm{c}}$ & \pm 11.9 \\
11 & 7 & $75.6^{\mathrm{d}}$ & \pm 12.0 \\
\hline
\end{tabular}

Those $\overline{\mathrm{x}}$ values with different superscripts are significantly different using $\operatorname{LSD}_{.05}=15.10$.

TABLE 5

Ammonia content $(\mathrm{mg} / \ell)$ of Cabernet Sauvignon grapes from 10 vineyards

\begin{tabular}{c|c|c|c}
\hline $\begin{array}{c}\text { Grower } \\
\text { (Vineyard) }\end{array}$ & Number of loads & $\overline{\mathrm{X}}$ & $\mathrm{S}$ \\
\hline 1 & 13 & $153.3^{\mathrm{a}}$ & \pm 15.6 \\
14 & 6 & $134.8^{\mathrm{a}, \mathrm{b}}$ & \pm 16.5 \\
15 & 5 & $134.4^{\mathrm{a}, \mathrm{b}}$ & \pm 14.3 \\
16 & 6 & $127.6^{\mathrm{b}}$ & \pm 25.3 \\
17 & 5 & $116.8^{\mathrm{b}, \mathrm{c}}$ & \pm 8.9 \\
18 & 5 & $105.8^{\mathrm{c}, \mathrm{d}}$ & \pm 36.9 \\
19 & 10 & $99.3^{\mathrm{c}, \mathrm{d}, \mathrm{e}}$ & \pm 12.6 \\
20 & 8 & $87.8^{\mathrm{d}, \mathrm{e}}$ & \pm 21.6 \\
21 & 6 & $87.5^{\mathrm{d}, \mathrm{e}}$ & \pm 7.2 \\
22 & 5 & $81.0^{\mathrm{e}}$ & \pm 9.1 \\
\hline
\end{tabular}

Those $\bar{x}$ values with different superscripts are significantly different using $\operatorname{LSD}_{.05}=19.11$ 
TABLE 6

Ammonia content $(\mathrm{mg} / \ell)$ of Clairette Blanche grapes from nine vineyards

\begin{tabular}{c|c|c|c}
\hline $\begin{array}{c}\text { Grower } \\
\text { (Vineyard) }\end{array}$ & Number of loads & $\overline{\mathrm{X}}$ & $\mathrm{S}$ \\
\hline 7 & 14 & $71.6^{\mathrm{a}}$ & \pm 7.0 \\
1 & 7 & $70.7^{\mathrm{a}}$ & \pm 7.4 \\
8 & 10 & $67.2^{\mathrm{a}}$ & \pm 17.2 \\
30 & 5 & $64.4^{\mathrm{a}}$ & \pm 5.8 \\
5 & 14 & $63.3^{\mathrm{a}}$ & \pm 16.2 \\
31 & 7 & $52.4^{\mathrm{b}}$ & \pm 10.3 \\
26 & 5 & $51.0^{\mathrm{b}}$ & \pm 4.2 \\
9 & 7 & $48.4^{\mathrm{b}}$ & \pm 7.6 \\
4 & 14 & $46.5^{\mathrm{b}}$ & \pm 6.0 \\
\hline
\end{tabular}

Those $\overline{\mathrm{x}}$ values with different superscripts are significantly different using $\mathrm{LSD}_{.05}=10.13$.

TABLE 7

Ammonia content $(\mathrm{mg} / \ell)$ of Chenin Blanc (steen) grapes from 16 vineyards

\begin{tabular}{c|c|c|c}
\hline $\begin{array}{c}\text { Grower } \\
\text { (Vineyards) }\end{array}$ & Number of loads & $\overline{\mathrm{X}}$ & $\mathrm{S}$ \\
\hline 36 & 20 & $78.2^{\mathrm{a}}$ & \pm 13.3 \\
26 & 11 & $73.9^{\mathrm{a}, \mathrm{b}}$ & \pm 21.9 \\
37 & 6 & $66.2^{\mathrm{a}, \mathrm{b}, \mathrm{c}}$ & \pm 8.0 \\
30 & 5 & $65.6^{\mathrm{b}, \mathrm{c}}$ & \pm 20.4 \\
38 & 5 & $64.8^{\mathrm{b}, \mathrm{c}}$ & \pm 14.7 \\
20 & 20 & $62.3^{\mathrm{c}, \mathrm{d}}$ & \pm 15.5 \\
5 & 18 & $60.4^{\mathrm{c}, \mathrm{d}, \mathrm{e}}$ & \pm 19.6 \\
2 & 14 & $59.9^{\mathrm{c}, \mathrm{d}, \mathrm{e}}$ & \pm 8.6 \\
16 & 6 & $58.5^{\mathrm{c}, \mathrm{d}, \mathrm{e}}$ & \pm 11.7 \\
39 & 5 & $57.8^{\mathrm{c}, \mathrm{d}, \mathrm{e}}$ & \pm 15.5 \\
3 & 20 & $54.6^{\mathrm{c}, \mathrm{d}, \mathrm{e}}$ & \pm 17.3 \\
29 & 15 & $52 .^{\mathrm{c}, \mathrm{d}, \mathrm{e}}$ & \pm 12.8 \\
4 & 9 & $51.7^{\mathrm{d}, \mathrm{e}}$ & \pm 6.1 \\
14 & 26 & $51.2^{\mathrm{e}}$ & \pm 17.2 \\
34 & 5 & $39.8^{\mathrm{e}, \mathrm{f}}$ & \pm 10.0 \\
11 & 8 & $35.1^{\mathrm{f}}$ & \pm 3.4 \\
\hline
\end{tabular}

Those $\bar{x}$ values with different superscripts are significantly different using $\operatorname{LSD}_{.05}=12.85$.

TABLE 8

Comparison of "high" ammonia" vineyards to viticultural conditions and treatments

\begin{tabular}{|c|c|c|c|c|c|c|c|c|c|c|c|c|c|c|c|}
\hline \multirow[b]{2}{*}{ Grower } & \multicolumn{13}{|c|}{ Cultivars } & \multicolumn{2}{|r|}{ Viticultural Aspects } \\
\hline & 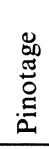 & 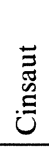 & 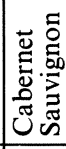 & 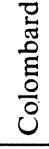 & 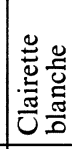 & 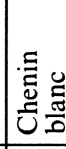 & 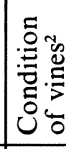 & 离 & "̄ & 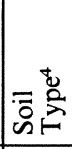 & 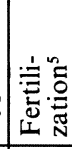 & 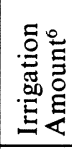 & $\frac{\pi}{2}$ & Trellis & Remarks \\
\hline 1 & $\sqrt{ }$ & & & & & & G & 12 & $\mathrm{D}$ & HA & 30 & $\begin{array}{r}0 \\
600\end{array}$ & 12 & 3 wire trellis & Devon Valley - Western slope. \\
\hline 1 & & & $\sqrt{ }$ & & & & G & 8 & $\mathrm{D}$ & HA & 30 & 600 & 8 & 3 wire trellis & Devon Valley - Western slope. \\
\hline 1 & & & & & $\sqrt{ }$ & & A & $15+$ & $\mathrm{D}$ & HA & 30 & 600 & 12 & 3 wire trellis & Devon Valley - Western slope. \\
\hline 2 & $\sqrt{ }$ & & & & & & G & 8 & $\mathbf{M}$ & LN & 25 & 500 & 12 & 3 wire trellis & Vlottenburg - Slight eastern slope. \\
\hline 2 & & & & $\sqrt{ }$ & & & A & 4 & S & LN & 20 & 500 & 8 & 3 wire trellis & Vlottenburg - Slight eastern slope. \\
\hline 3 & & $\sqrt{ }$ & & & & & A & 15 & $\mathbf{M}$ & LN & 30 & $\begin{array}{l}100 \\
600\end{array}$ & 10 & untrellised & Stellenbosch - Northern slope. \\
\hline 5 & $\sqrt{ }$ & & & & & & G & 8 & $\mathrm{D}$ & HA & 30 & 600 & 12 & 3 wire trellis & Devon Valley. High altitude - cool climate. \\
\hline 7 & & $\sqrt{ }$ & & & & & A & 15 & $\mathbf{M}$ & HA & 20 & 550 & 10 & untrellised & Koelenhof - Northern slope. \\
\hline 7 & & & $\sqrt{ }$ & & & & A & 12 & M & HA & 20 & $\begin{array}{r}50 \\
550\end{array}$ & 6 & 3 wire trellis & Koelenhof - Western slope. \\
\hline 7 & & & & & $\sqrt{ }$ & & A & 15 & M & HA & 20 & $\begin{array}{r}50 \\
550\end{array}$ & 12 & 3 wire trellis & Koelenhof - Western slope. \\
\hline 8 & & & & & $\sqrt{ }$ & & A & 5 & M & HA & 20 & $\begin{array}{l}100 \\
600\end{array}$ & 12 & 3 wire trellis & Simonsberg. \\
\hline 14 & & & $\sqrt{ }$ & & & & G & 9 & $\mathbf{M}$ & HA & 30 & $\begin{array}{l}100 \\
500\end{array}$ & 10 & 3 wire trellis & Koelenhof. (Chicken manure \& fertilizer). \\
\hline 15 & & & $\sqrt{ }$ & & & & G & 8 & D & HA & 20 & 600 & 8 & 3 wire trellis & Devon Valley - adjoining. \\
\hline 16 & & & $\sqrt{ }$ & & & & G & 7 & D & HA & 20 & 600 & 8 & 3 wire trellis & Devon Valley - adjoining. \\
\hline 24 & & & & & & $\sqrt{ }$ & A & 4 & $\mathrm{~S}$ & LN & 20 & 500 & 13 & 3 wire trellis & Young vineyard overcropped. \\
\hline 28 & & & & & & $\sqrt{ }$ & A & 12 & M & LN & 20 & $\begin{array}{r}50 \\
500\end{array}$ & 10 & untrellised & Bottelary. \\
\hline 32 & & & & & $\sqrt{ }$ & & A & 25 & D & HA & 20 & 750 & 8 & untrellised & Simonsberg - Western slope. \\
\hline 36 & & & & & & $\sqrt{ }$ & G & 4 & D & LA & 30 & $75 \stackrel{?}{?}$ & 16 & 6 wire trellis & $\begin{array}{l}\text { Jonkershoek - low lying dark alluvial soil } \\
\text { Drip irrigated - overcropped. }\end{array}$ \\
\hline
\end{tabular}

${ }^{1}$ More than $\frac{1}{2}$ standard deviation above the mean value of that cultivar. ${ }_{5}^{5}$ as $\mathrm{kg} \mathrm{N} / \mathrm{ha}$

${ }^{2} \mathrm{G}=$ good; $\mathrm{A}=$ average $\mathbf{P}=$ poor.

${ }^{3} \mathrm{D}=$ deep $; \mathrm{M}=$ medium $; \mathrm{S}=$ shallow

${ }^{4} \mathrm{H}=$ heavy $; \mathrm{L}=$ light $; \mathrm{A}=$ acid $; \mathrm{N}=$ neutral.

${ }^{6}$ as applied (upper figure) and rainfall (lower figure) in $\mathrm{mm}$. 7 as tonnes/ha. 
TABLE 9

Comparison of "low ${ }^{1}$ ammonia" vineyards to viticultural conditions and treatments

\begin{tabular}{|c|c|c|c|c|c|c|c|c|c|c|c|c|c|c|c|c|}
\hline \multirow[b]{2}{*}{ Grower } & \multicolumn{14}{|c|}{ Cultivars } & \multicolumn{2}{|r|}{ Viticultural Aspects } \\
\hline & 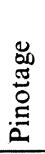 & 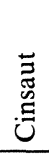 & 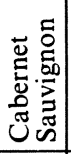 & $\begin{array}{l}\text { 뮤 } \\
\text { है } \\
\text { 응 } \\
0\end{array}$ & 苐 & 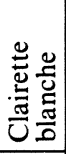 & 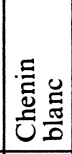 & 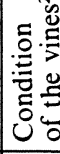 & 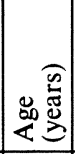 & : & 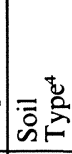 & 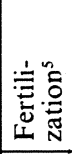 & 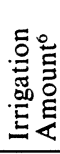 & $\stackrel{\frac{\pi}{0}}{\frac{\pi}{2}}$ & Trellis & Remarks \\
\hline 4 & & & & & & $\sqrt{ }$ & & A & 6 & D & HA & $0-15$ & $\begin{array}{r}0 \\
550\end{array}$ & 12 & 1 wire & Low fertilization, overcrop. \\
\hline 4 & $\sqrt{ }$ & & & & & & & G & 8 & D & HA & $0-15$ & 550 & 5 & 1 wire & High altitude 3.5 slope. \\
\hline 4 & & & $\sqrt{ }$ & & & & & A & 15 & D & HA & $0-15$ & 550 & 12 & 1 wire & Half long pruning. \\
\hline 6 & $\sqrt{ }$ & & & & & & & A & 10 & $\mathbf{M}$ & LN & 20 & $\begin{array}{l}100 \\
500\end{array}$ & 15 & 4 wire veranda & Overcropping (Helderberg). \\
\hline 9 & & & $\sqrt{ }$ & & & & & $\mathbf{P}$ & \pm 15 & $S$ & LN & 0 & 500 & 4 & 1 wire & Neglected. Oidium. \\
\hline 9 & & & & & & $\sqrt{ }$ & & $\mathbf{P}$ & \pm 20 & S & LN & 0 & 500 & 6 & untrellised & Neglected. Oidium. \\
\hline 11 & & $\sqrt{ }$ & & & & & & $\mathbf{P}$ & \pm 20 & $\mathbf{S}$ & LN & \pm 15 & $\begin{array}{l}100 \\
450\end{array}$ & 6 & untrellised & Vines very old. \\
\hline 11 & & & $\sqrt{ }$ & & & & & P & 8 & $S$ & LN & \pm 15 & $\begin{array}{l}150 \\
450\end{array}$ & 5 & 3 wire & Spacing $1 \mathrm{~m} \times 1 \mathrm{~m}$ straw mulch. \\
\hline 11 & & & & & & & $\sqrt{ }$ & A & 12 & $\mathbf{S}$ & LN & \pm 15 & $\begin{array}{l}100 \\
450\end{array}$ & 10 & 3 wire & Poor sandy soil. Southern slope. \\
\hline 12 & & $\sqrt{ }$ & & & & & & $\mathbf{P}$ & $20+$ & S & LN & 0 & 600 & 6 & untrellised & Cane pruned. \\
\hline 13 & & $\sqrt{ }$ & & & & & & $\mathbf{P}$ & $15+$ & S & LN & 0 & 500 & 5 & untrellised & Bad management. \\
\hline 13 & & & & & & & $\sqrt{ }$ & A & $15+$ & $\mathbf{S}$ & LN & 0 & 500 & 6 & untrellised & Bad management. \\
\hline 20 & & & $\sqrt{ }$ & & & & & A & 12 & $\mathbf{M}$ & HA & 20 & 500 & 6 & 3 wire & Poor selected vines. \\
\hline 20 & & & & & $\sqrt{ }$ & & & A & 9 & $\mathbf{M}$ & HA & 20 & 500 & 6 & 3 wire & Poorly pruned. Overripe. \\
\hline 21 & & & $\sqrt{ }$ & & & & & A & 8 & $\mathbf{M}$ & LN & $?$ & 500 & 6 & 3 wire & Oidium on leaves. \\
\hline 25 & & & $\sqrt{ }$ & & & & & A & 7 & $\mathbf{M}$ & LN & $?$ & $\begin{array}{l}100 \\
500\end{array}$ & 5 & 3 wire & \\
\hline 26 & & & & & & $\sqrt{ }$ & & A & $15+$ & D & HA & 10 & 600 & 10 & untrellised & Bad management. \\
\hline 29 & & & & $\sqrt{ }$ & & & & $\mathbf{P}$ & 12 & $\mathbf{M}$ & HA & 20 & $\begin{array}{l}100 \\
600\end{array}$ & 12 & 3 wire & Overcropping. Poor drainage. \\
\hline 31 & & & & & & $\sqrt{ }$ & & A & $15+$ & $\mathbf{M}$ & LN & 15 & $\begin{array}{r}0 \\
600\end{array}$ & 12 & 3 wire & \\
\hline 33 & & & & & & $\sqrt{ }$ & & A & 12 & $\mathbf{M}$ & LN & 20 & 700 & 8 & untrellised & Southern slope. \\
\hline 34 & & & & & & $\sqrt{ }$ & & A & $15+$ & $\mathbf{M}$ & LN & 0 & 500 & 7 & 3 wire & New owner. Neglected. \\
\hline 34 & & & & & & & $\sqrt{ }$ & A & $15+$ & $\mathbf{M}$ & LN & 0 & 500 & 8 & 3 wire & New owner. Neglected. \\
\hline 35 & & & & & & $\sqrt{ }$ & & $\mathbf{P}$ & $15+$ & $\mathbf{M}$ & LN & 20 & 600 & 8 & untrellised & Bad management. \\
\hline 45 & & & & & & & $\sqrt{ }$ & $\mathbf{P}$ & $15+$ & $\mathbf{M}$ & LN & 15 & 750 & 6 & 3 wire & Poorly drained dark alluvial soil. \\
\hline
\end{tabular}

${ }^{1}$ More than $\frac{1}{2}$ standard deviation below the mean value of that cultivar. ${ }^{5}$ as $\mathrm{kg} \mathrm{N} / \mathrm{ha}$.

${ }^{2} \mathrm{G}=$ good; $\mathrm{A}=$ average; $\mathrm{P}=$ poor.

${ }^{3} \mathrm{D}=$ deep; $\mathrm{M}=$ medium $; \mathrm{S}=$ shallow

${ }^{6}$ as applied (upper figure) and rainfall (lower figure) in $\mathrm{mm}$.

${ }^{4} \mathrm{H}=$ heavy; $\mathrm{L}=$ light; $\mathrm{A}=$ acid $; \mathrm{N}=$ neutral.

7 as tonnes/ha. 
vineyards generally 12 years old or older. Most of the vineyards in good condition were on deep soils, while the poor ones were on shallow soils, and the average ones mostly on medium soils. The good condition vines were on the heavier and acid soils, and the poor ones on the light neutral soils. All the high ammonia vineyards had from 20 to $30+\mathrm{kg} \mathrm{N} /$ ha added, while the low ammonia vineyards generally had from $0-20 \mathrm{~kg} \mathrm{~N} /$ ha added. Crops were somewhat lower for the low ammonia vineyards. Trellising differences were not apparent. The remarks indicate some overcropping in both groups (two for the high ammonia vineyards and four for the low ammonia vineyards). The main adverse notes on the low ammonia vineyards are: neglect, oidium, and bad management.

A large group of growers fall into the intermediate group and are not categorized other than having medium ammonia vineyards and probably would fall somewhere between two groups as far as their vineyard conditions are concerned.

The use of ${ }^{\circ} \mathrm{Brix}, \mathrm{pH}$ and $\mathrm{NH}_{3}$ measurements of fruit seems to be an easy way of judging the potential quality of the fruit from a given area and cultivar. This can be backed up by vineyard inspection and experimental fermentations for wine quality and fermentability. The total acidity and $\mathrm{Ph}$ are indicators which can also be considered primarily to indicate the need for acid addition at the crusher. The ${ }^{\circ} \mathrm{Brix}$ is a necessary criterium because of the change in ammonia content with maturity. As long as the ${ }^{\circ} \mathrm{Brix}$ is in the normal range $20-23,5^{\circ}$ Brix the ammonia value is consistent with the data furnished (Ough, 1969). However, early picked or $25+{ }^{\circ}$ Brix fruit should not be judged by these standards.

Further data to support the validity of these studies are certainly desirable. Variations in seasons can be a factor in the nitrogen uptake in the vines and the consequent ammonia content of the grape. However, the relative differences between vineyards should not change.

The purpose of such a study as this is to determine which vineyards produce fruit which are valuable, as far as making sound and good quality wines is concerned. It is not a short-range project to screen individual loads of grapes. Further studies relating the quality of wines made from the individual vineyards to the analysis should be the follow-up project.

\section{LITERATURE CITED}

AGENBACH, W. A. 1977. A study of must nitrogen content in relation to incomplete fermentations, yeast productions and fermentation activity. Proc. S. African Soc. Enol. Vitic, Cape Town 21-22 Nov. p. 66-88.

AMERINE, M. A., \& ROESSLER, E. B. 1958. Field testing of grape maturity. Hilgardia 28, 93-114.

BELL A. A., OUGH, C. S., \& KLIEWER, W. M. 1979. Effects on must and wine composition, rate of fermentation and wine quality of nitrogen fertilization of Vitis vinifera var Thompson Seedless grapes. Am. J. Enol. Vitic. 30, 124-129.

BERG, H. W. \& MARSH, G. L. 1954. Sampling deliveries of grapes on a representative basis. Food Techol. 8, 104-108.

McWILLIAM, D. J. \& OUGH, C. S. 1974. Measurement of ammonia in musts and wines using a selective electrode. Am.J. Enol. Vitic. 25, 67-72.

OUGH, C. S. 1969. Unpublished data

OUGH, C. S. 1969. Ammonia content of California grapes. Am. J. Enol. Vitic. 20, 213-220.

OUGH, C. S. \& BELL, A. A. 1980. Effects of nitrogen fertilization of grapevine on amino acid metabolism and higher-alcohol formation during grape juice fermentation. Am. J. Enol. Vitic. 31, 122-123.

OUGH, C.S. \& KUNKEE, R. E. 1968. Fermentation rate of grape juice $\mathrm{V}$. Biotin content and its effect on alcoholic fermentation rate. Appl. Microbiol. 16, 572-576.

OUGH, C. S. \& LEE, T. H. 1981. The effects of vineyard nitrogen fertilization level on the formation of some fermentation esters. Am J.. Enol. Vitic. 32, 125-127.

OUGH, C. S. \& SINGLETON, V. L. 1968. Wine quality production from juice Brix/acid ratio and associated compositional changes for "White Riesling" and "Cabernet Sauvignon". Am. J. Enol. Vitic. 19, 129-138.

PUISSANT, A., HERVE, J. \& TENTRY, M. 1960. Etude sur la variation de la tenure en $\mathrm{NH}^{+}{ }_{4}, \mathrm{~K}^{+}, \mathrm{Ca}{ }^{++}$and $\mathrm{Mg}^{++}$de quelques raisins au cours de la maturation. Ann. Technol. Agric. 4, 321-330.

SNEDECOR, G. W. 1956. Statistical Methods. 5th Ed. 534 p. The Iowa State University Press. Ames, Iowa, U.S.A.

VOS, P. J. A., ZEEMAN, W. \& HEYMANN, H. 1979. The effect on wine quality of diammonuim phospahte additions to musts. J. South African Soc. Enol. Vitic. 1, 87-104. 